Egyptian Journal of Aquatic Biology \& Fisheries

Zoology Department, Faculty of Science,

Ain Shams University, Cairo, Egypt.

ISSN $1110-6131$

Vol. 24(5): 237 - 248 (2020)

www.ejabf.journals.ekb.eg

\title{
Chromosomal Formula of Four Marine Fish Species from the Mediterranean Sea in Egypt
}

\author{
Ali H. Abu Almaaty*, Hala E. Abd-Alaty, Osama A. Abbas and \\ Mohamed K. Hassan \\ Zoology Department, Faculty of Science, Port Said University, Port Said, Egypt. \\ "Corresponding Author: ali_zoology_2010@yahoo.com
}

\section{ARTICLE INFO}

Article History:

Received: July 15, 2020

Accepted: July 22, 2020

Online: July 25, 2020

Keywords:

Genetics

Fish

Karyotypes

Chromosomes

Perciformes
ABSTRACT

The karyological analysis is an important tool for the detection of biodiversity; it is also used for measuring biodiversity evolutionary aspects. Several families of Perciformes showed a remarkable degree of chromosomal conservation $(2 \mathrm{n}=48, \mathrm{FN}=48)$. The present study was aimed to characterize cytogenetically the chromosomal formula, chromosome numbers and karyotypes of four fish species of order Perciformes; (Argyrosomus regius, Pomadasys stridens, Sparus aurata and Dicentrarchus labrax) collected from the Mediterranean Sea in Port Said. The Mitotic chromosomal spreads illustrated that all chromosomes of these four species are acrocentric chromosomes, the chromosomal formula for all four fish species was $48 \mathrm{a}$ and the fundamental number for all four fish species is $(\mathrm{FN}=48)$. The relative length ranged from $1.82 \%$ to $5.61 \%$ in Argyrosomus regius $2.73 \%$ to $5.61 \%$ in Pomadasys stridens, $1.91 \%$ to $5.83 \%$ in Sparus aurata and in Dicentrarchus labrax it ranged from $2.48 \%$ to $5.59 \%$. All species had centromeric index equal zero and arm ratio equal $\infty$.

\section{INTRODUCTION}

More than half of known vertebrates are fishes, the recognized fish species are more than 32,000 species (Eschmeyer and Fong. 2014). The different fish species show a variety of behavior, morphology and habitat (Nelson, 2006). Fishes in marine habitat exhibit a little diversity in chromosome numbers and the karyotype formula among different species; this is because of the absence of geographical barriers that is found in fresh water habitat hindering the gene flow among populations. So, a great variation in chromosome numbers is clearly observed in fish species of freshwater habitat and absent in that is live in marine habitat (Bloom, et al., 2013).

Cytogenetic data of fishes are very little; they only represent approximately $10.7 \%$ of all recognized species in the world (Nirchio, et al., 2014). Karyological analysis is one of cytogenetic techniques; it aims to study the number, morphology and size of chromosomes for a species. Mitotic chromosomal spreads are used for this analysis (Shalaby, et al., 2020). 
Karyotyping is a process by which we can establish the chromosomes number, chromosomes length, centrosomes position, banding pattern and sex chromosomes differences. Karyotyping is a part of cytogenetic (King, et al., 2006). Standardized staining procedures are used in karyotype preparations (O'Connor, 2008).

There is a great variation in chromosome number among marine fishes; however, there are many groups, such as Perciformes that contain more than 700 marine species including many marine teleosts that have an economic importance and little chromosome divergence. Previous studies illustrated that the karyotype of approximately $60 \%$ of fish species consists of acrocentric chromosomes such as Sciaenidae, Blenniidae, Sparidae, some pomacentridae, Serranidae, Nototheniidae, Channichthyidae (Neto, et al., 2011).

Order perciformes is a large order including the largest number of teleostei species. Brum, (1995) stated that about 420 species of 50 perciformes families were karyologically studied and $67 \%$ of the studied species had a diploid chromosome number equal 48 chromosomes, $30 \%$ had $2 n$ less than 48 chromosomes and $3 \%$ had $2 n$ more than 48 chromosomes. Nirchio et al., (2014) studied the karyotype of 70 marine fish species , twenty five of them representing about $35.71 \%$ of the total species had 48 acrocentric chromosomes and approximately $60 \%$ of all studied species had a diploid chromosome number equal to 48 chromosomes.

Because of the scarcity of chromosomal evolution reports on fish species of order percifomes in Egypt, the aim of this study is to provide new chromosomal data for four fish species (Argyrosomus regius, Pomadasys stridens, Sparus aurata and Dicentrarchus labrax) collected from the Mediterranean Sea in Port Said in Egypt and to compare these results to previous published chromosomal data, in order to correlate chromosomal rearrangements during the evolutionary history of the order Perciformes.

\section{MATERIALS AND METHODS}

Cytogenetical investigations were performed on four fish species (Argyrosomus regius, Pomadasys stridens, Sparus aurata and Dicentrarchus labrax) collected from Mediterranean Sea in Port Said in Egypt.

\section{Cytogenetical technique}

Mitotic chromosomes were obtained from kidney, gills and spleen as described by (Netto et al., 2007). Fish samples were kept in appropriate aquarium then they were injected with $0.05 \%$ colchicine ( $1 \mathrm{ml}$ per $100 \mathrm{~g}$ fish weight). After approximately two hours, samples were dissected and then tissues of kidney, gills and spleen were preserved in a hypotonic solution $(0.56 \% \mathrm{KCL})$ for approximately one hour. Fixation of the tissues was performed using a mixture of ethanol and glacial acetic acid (3:1); tissues were preserved in the fixative for twenty minutes. This step was repeated three times.

The fixed tissues were squashed in $60 \%$ glacial acetic acid forming a cellular suspension. Dropping of three droplets of the cellular suspension on a microscopic slide. Passing the slides over a flame and then dried on air. Staining of slides for approximately one hour using 5\% giemsa stain was performed.

\section{Chromosomal analysis and karyotyping:}

Examination of the slides under light microscope using x10 or x15 eyepieces, with x100 objectives and a good chromosomal spread were photographed for further karyotyping. According to (Molina et al., 2012\&2013), chromosomes were classified in 
the present study. Arm ratio (AR), Relative length (RL) and centromeric index (CI) were calculated.

\section{RESULTS}

The karyotype of four fish species under the present study showed that the chromosomes of those species are all acrocentric chromosomes and Ideogrames of chromosomes for all fish species were constructed in respect to relative length (Figures 2, 4, 6 and 8).

\section{Argyrosomus regius}

This species belongs to family scianidae. The photographed spreads of cells of this species (fig. 1$)$ and karyotype showed the diploid chromosome number $(2 n=48)$ and all were acrocentric chromosomes, as illustrated in (fig.1). The relative length of these chromosomes ranged from $1.82 \%$ to $5.61 \%$, centromeric indices were equal to zero and arm ratio of $\infty$ as illustrated in Table (1).

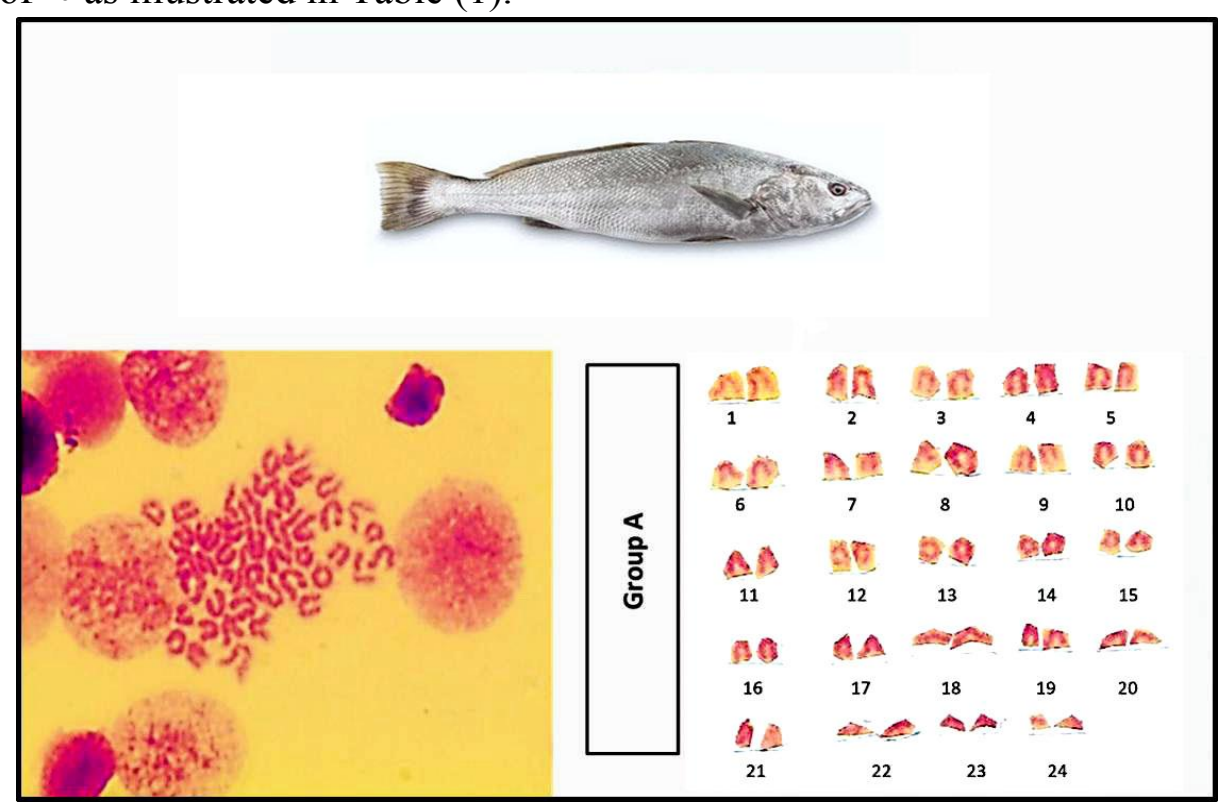

Fig. 1. A coloured photograph, chromosomes spread and karyotype of Argyrosomus regius.

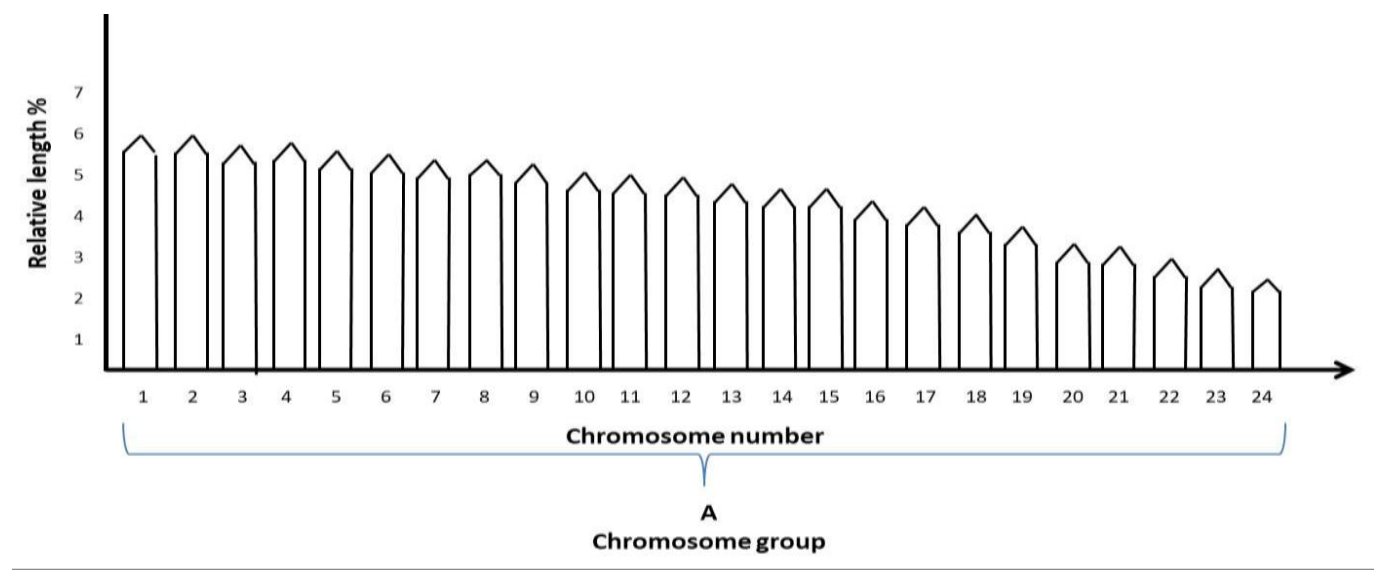

Fig. 2. Idiogram of chromosomes of Argyrosomus regius which constructed in respect to relative length. 
Ali H. Abu Almaaty et al., 2020

Table 1. Average of ten spreads of chromosomes measurements and classification of Argyrosomus regius.

\begin{tabular}{|c|c|c|c|c|c|c|c|c|c|}
\hline \multirow{3}{*}{ 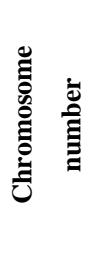 } & \multicolumn{3}{|c|}{ Chromosome length } & \multicolumn{3}{|c|}{ Relative length } & \multirow{2}{*}{$\begin{array}{l}\text { Arm } \\
\text { ratio }\end{array}$} & \multirow{2}{*}{$\begin{array}{c}\text { Centromeric } \\
\text { Index \% }\end{array}$} & \multirow{3}{*}{ 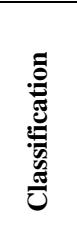 } \\
\hline & Long arm & $\begin{array}{l}\text { Short } \\
\text { arm }\end{array}$ & Total & Long arm & $\begin{array}{c}\text { Short } \\
\text { arm }\end{array}$ & Total & & & \\
\hline & Mean \pm S.D & $\begin{array}{c}\text { Mean } \pm \\
\text { S.D }\end{array}$ & Mean \pm S.D & Mean \pm S.D & $\begin{array}{c}\text { Mean } \pm \\
\text { S.D }\end{array}$ & Mean \pm S.D & $\begin{array}{l}\text { Mean } \pm \\
\text { S.D }\end{array}$ & Mean \pm S.D & \\
\hline 1 & $0.68 \pm 0.05$ & $\overline{0.0}$ & $0.68 \pm 0.05$ & $5.61 \pm 0.04$ & 0.0 & $5.61 \pm 0.04$ & $\infty$ & 0.0 & Acro. \\
\hline 2 & $0.67 \pm 0.05$ & 0.0 & $0.67 \pm 0.05$ & $5.53 \pm 0.04$ & 0.0 & $5.53 \pm 0.04$ & $\infty$ & 0.0 & Acro. \\
\hline 3 & $0.66 \pm 0.04$ & 0.0 & $0.66 \pm 0.04$ & $5.45 \pm 0.04$ & 0.0 & $5.45 \pm 0.04$ & $\infty$ & 0.0 & Acro. \\
\hline 4 & $0.65 \pm 0.04$ & 0.0 & $0.65 \pm 0.04$ & $5.36 \pm 0.03$ & 0.0 & $5.36 \pm 0.03$ & $\infty$ & 0.0 & Acro. \\
\hline 5 & $0.64 \pm 0.04$ & 0.0 & $0.64 \pm 0.04$ & $5.28 \pm 0.05$ & 0.0 & $5.28 \pm 0.05$ & $\infty$ & 0.0 & Acro. \\
\hline 6 & $0.62 \pm 0.03$ & 0.0 & $0.62 \pm 0.03$ & $5.12 \pm 0.03$ & 0.0 & $5.12 \pm 0.03$ & $\infty$ & 0.0 & Acro. \\
\hline 7 & $0.61 \pm 0.05$ & 0.0 & $0.61 \pm 0.05$ & $5.03 \pm 0.03$ & 0.0 & $5.03 \pm 0.03$ & $\infty$ & 0.0 & Acro. \\
\hline 8 & $0.60 \pm 0.03$ & 0.0 & $0.60 \pm 0.03$ & 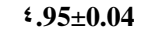 & 0.0 & $5.95 \pm 0.04$ & $\infty$ & 0.0 & Acro. \\
\hline 9 & $0.58 \pm 0.04$ & 0.0 & $0.58 \pm 0.04$ & $4.78 \pm 0.03$ & $\mathbf{0 . 0}$ & $4.78 \pm 0.03$ & $\infty$ & 0.0 & Acro. \\
\hline 10 & $0.57 \pm 0.04$ & 0.0 & $0.57 \pm 0.04$ & $4.70 \pm 0.04$ & 0.0 & $4.70 \pm 0.04$ & $\infty$ & 0.0 & Acro. \\
\hline 11 & $0.56 \pm 0.05$ & 0.0 & $0.56 \pm 0.05$ & $4.62 \pm 0.04$ & 0.0 & $4.62 \pm 0.04$ & $\infty$ & 0.0 & Acro. \\
\hline 12 & $0.55 \pm 0.05$ & 0.0 & $0.55 \pm 0.05$ & $4.54 \pm 0.05$ & 0.0 & $4.54 \pm 0.05$ & $\infty$ & 0.0 & Acro. \\
\hline 13 & $0.53 \pm 0.03$ & $\mathbf{0 . 0}$ & $0.53 \pm 0.03$ & $4.37 \pm 0.05$ & 0.0 & $4.37 \pm 0.05$ & $\infty$ & $\mathbf{0 . 0}$ & Acro. \\
\hline 14 & $0.51 \pm 0.02$ & 0.0 & $0.51 \pm 0.02$ & $4.21 \pm 0.03$ & 0.0 & $4.21 \pm 0.03$ & $\infty$ & 0.0 & Acro. \\
\hline 15 & $0.50 \pm 0.05$ & 0.0 & $0.50 \pm 0.05$ & $4.13 \pm 0.04$ & 0.0 & $4.13 \pm 0.04$ & $\infty$ & 0.0 & Acro. \\
\hline 16 & $0.47 \pm 0.05$ & 0.0 & $0.47 \pm 0.05$ & $3.88 \pm 0.04$ & $\mathbf{0 . 0}$ & $3.88 \pm 0.04$ & $\infty$ & 0.0 & Acro. \\
\hline 17 & $0.45 \pm 0.04$ & 0.0 & $0.45 \pm 0.04$ & $3.71 \pm 0.05$ & 0.0 & $3.71 \pm 0.05$ & $\infty$ & 0.0 & Acro. \\
\hline 18 & $0.42 \pm 0.04$ & 0.0 & $0.42 \pm 0.04$ & $3.47 \pm 0.05$ & 0.0 & $3.47 \pm 0.05$ & $\infty$ & $\mathbf{0 . 0}$ & Acro. \\
\hline 19 & $0.38 \pm 0.03$ & 0.0 & $0.38 \pm 0.03$ & $3.14 \pm 0.03$ & 0.0 & $3.14 \pm 0.03$ & $\infty$ & 0.0 & Acro. \\
\hline 20 & $0.36 \pm 0.03$ & 0.0 & $0.36 \pm 0.03$ & $2.97 \pm 0.03$ & $\mathbf{0 . 0}$ & $2.97 \pm 0.03$ & $\infty$ & 0.0 & Acro. \\
\hline 21 & $0.35 \pm 0.03$ & 0.0 & $0.35 \pm 0.03$ & $2.89 \pm 0.05$ & 0.0 & $2.89 \pm 0.05$ & $\infty$ & 0.0 & Acro. \\
\hline 22 & $0.30 \pm 0.04$ & 0.0 & $0.30 \pm 0.04$ & $2.48 \pm 0.04$ & 0.0 & $2.48 \pm 0.04$ & $\infty$ & 0.0 & Acro. \\
\hline 23 & $0.25 \pm 0.05$ & $\mathbf{0 . 0}$ & $0.25 \pm 0.05$ & $2.06 \pm 0.04$ & 0.0 & $2.06 \pm 0.04$ & $\infty$ & 0.0 & Acro. \\
\hline 24 & $0.22 \pm 0.05$ & 0.0 & $0.22 \pm 0.05$ & $1.82 \pm 0.03$ & 0.0 & $1.82 \pm 0.03$ & $\infty$ & 0.0 & Acro. \\
\hline Sum. & & & $12.12 \pm 0.03$ & & & & & & \\
\hline
\end{tabular}

\section{Dicentrarchus labrax}

This species belongs to family moronidae. The photographed spreads of cells of this species (Fig.3) and karyotype showed the diploid chromosome number $(2 n=48)$ and all were acrocentric chromosomes, as illustrated in (Fig.3). The relative length of these chromosomes ranged from $2.48 \%$ to $5.95 \%$, centromeric indices were equal to zero and arm ratio of $\infty$ as shown in Table (2). 


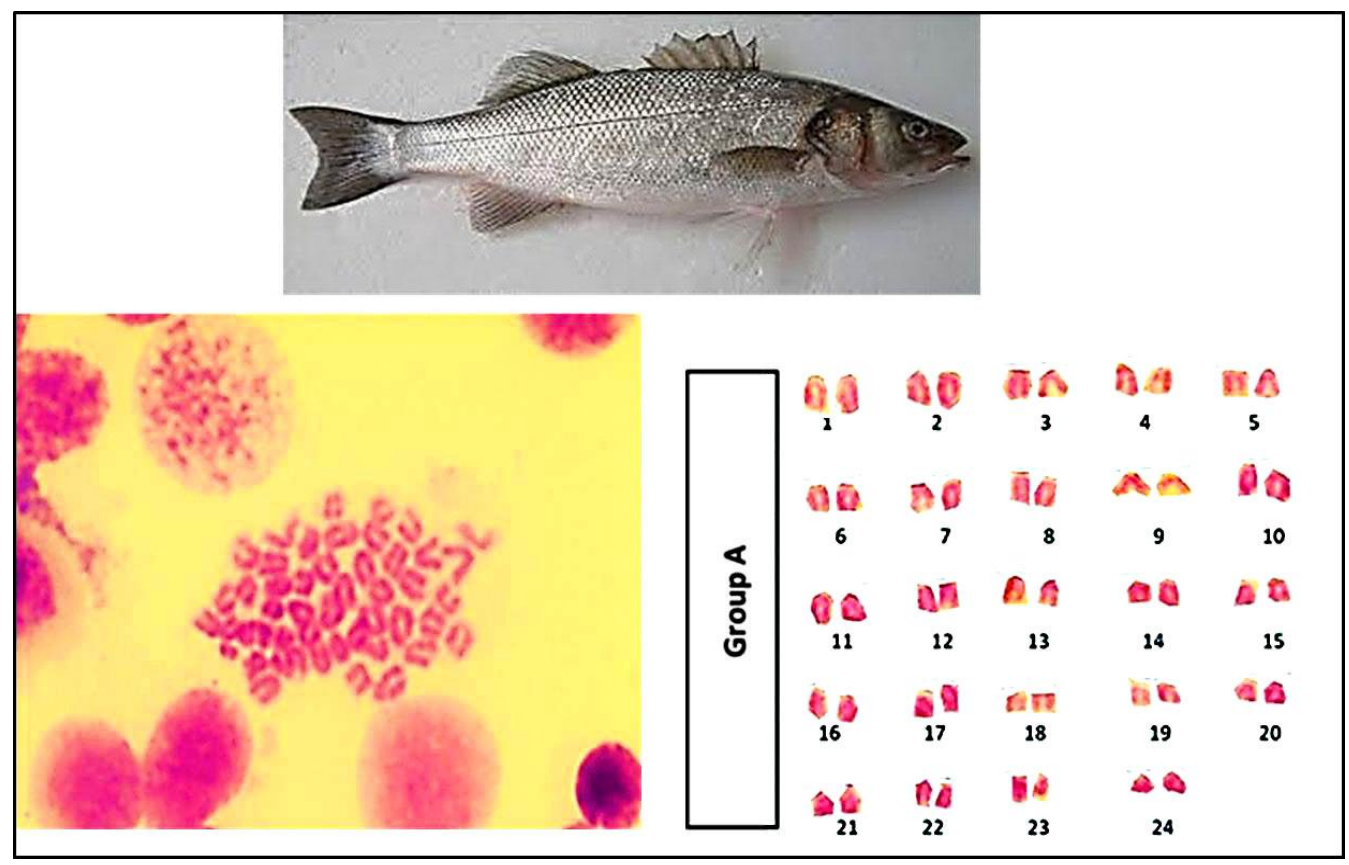

Fig. 3. A coloured photograph, chromosomes spread and karyotype of Dicentrarchus labrax.

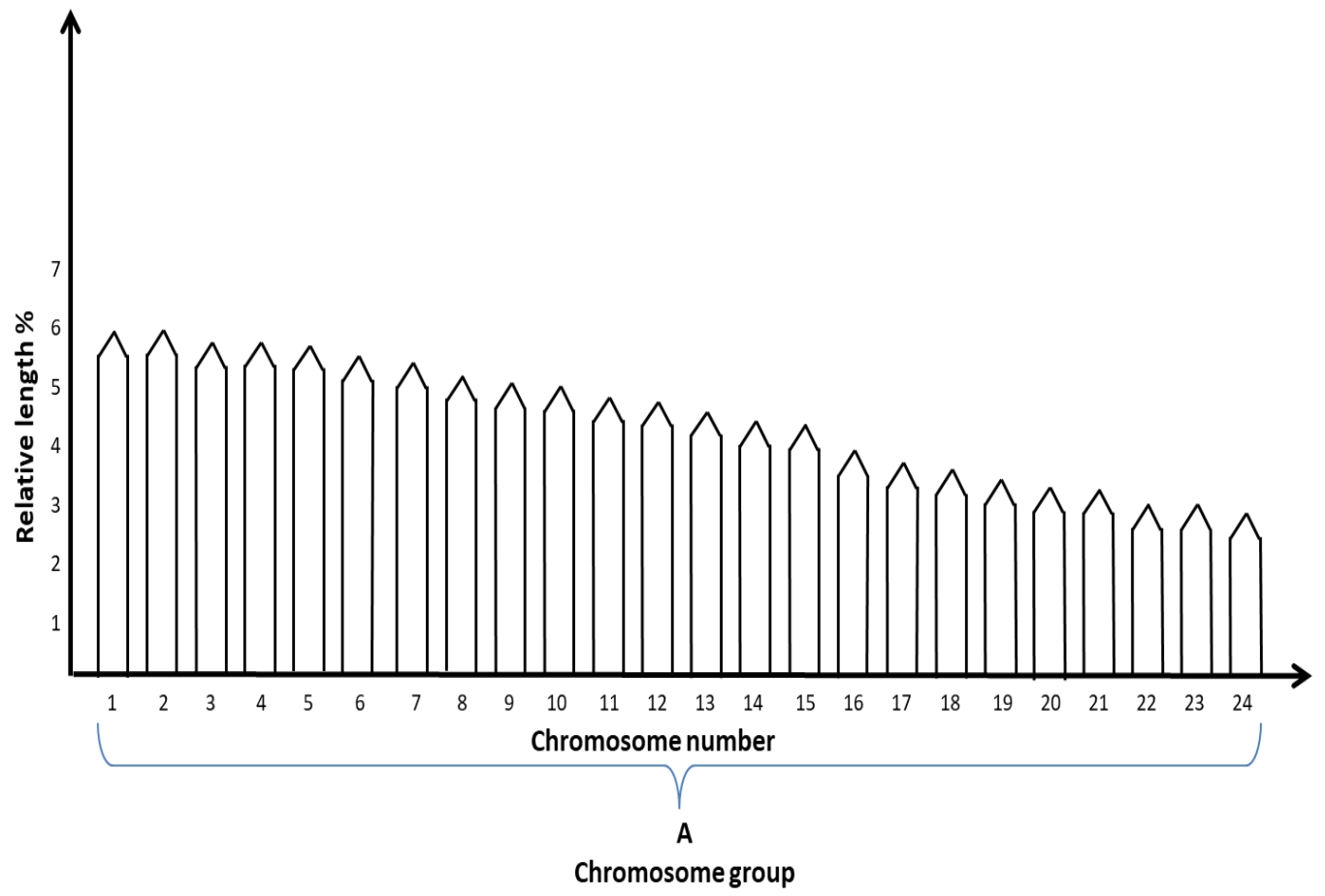

Fig. 4. Idiogram of chromosomes of Dicentrarchus labrax which constructed in respect to relative length. 
Table 2. Average of ten spreads of chromosomes measurements and classification of Dicentrarchus labrax.

\begin{tabular}{|c|c|c|c|c|c|c|c|c|c|}
\hline \multirow{3}{*}{ 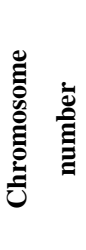 } & \multicolumn{3}{|c|}{ Chromosome length } & \multicolumn{3}{|c|}{ Relative length } & \multirow{2}{*}{$\begin{array}{l}\text { Arm } \\
\text { ratio }\end{array}$} & \multirow{2}{*}{$\begin{array}{c}\text { Centromeric } \\
\text { Index } \%\end{array}$} & \multirow{3}{*}{ 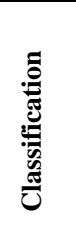 } \\
\hline & Long arm & $\begin{array}{l}\text { Short } \\
\text { arm }\end{array}$ & Total & Long arm & $\begin{array}{l}\text { Short } \\
\text { arm }\end{array}$ & Total & & & \\
\hline & Mean \pm S.D & $\begin{array}{c}\text { Mean } \pm \\
\text { S.D }\end{array}$ & Mean \pm S.D & Mean \pm S.D & $\begin{array}{c}\text { Mean } \pm \\
\text { S.D }\end{array}$ & Mean \pm S.D & $\begin{array}{c}\text { Mean } \pm \\
\text { S.D }\end{array}$ & Mean \pm S.D & \\
\hline $\mathbf{1}$ & $0.70 \pm 0.04$ & 0.0 & $0.70 \pm 0.04$ & $5.59 \pm 0.03$ & 0.0 & $5.59 \pm 0.03$ & $\infty$ & 0.0 & Acro. \\
\hline 2 & $0.69 \pm 0.04$ & 0.0 & $0.69 \pm 0.04$ & $5.51 \pm 0.03$ & 0.0 & $5.51 \pm 0.03$ & $\infty$ & 0.0 & Acro. \\
\hline 3 & $0.68 \pm 0.04$ & $\mathbf{0 . 0}$ & $0.68 \pm 0.04$ & $5.43 \pm 0.04$ & 0.0 & $5.43 \pm 0.04$ & $\infty$ & 0.0 & Acro. \\
\hline 4 & $0.67 \pm 0.05$ & 0.0 & $0.67 \pm 0.05$ & $5.35 \pm 0.03$ & 0.0 & $5.35 \pm 0.03$ & $\infty$ & 0.0 & Acro. \\
\hline 5 & $0.66 \pm 0.05$ & 0.0 & $0.66 \pm 0.05$ & $5.27 \pm 0.04$ & $\overline{0.0}$ & $5.27 \pm 0.04$ & $\infty$ & 0.0 & Acro. \\
\hline 6 & $0.64 \pm 0.03$ & 0.0 & $0.64 \pm 0.03$ & $5.11 \pm 0.05$ & 0.0 & $5.11 \pm 0.05$ & $\infty$ & 0.0 & Acro. \\
\hline 7 & $0.62 \pm 0.04$ & 0.0 & $0.62 \pm 0.04$ & $4.95 \pm 0.05$ & 0.0 & $4.95 \pm 0.05$ & $\infty$ & 0.0 & Acro. \\
\hline 8 & $0.61 \pm 0.04$ & 0.0 & $0.61 \pm 0.04$ & $4.87 \pm 0.05$ & 0.0 & $4.87 \pm 0.05$ & $\infty$ & 0.0 & Acro. \\
\hline 9 & $0.60 \pm 0.03$ & 0.0 & $0.60 \pm 0.03$ & $4.79 \pm 0.05$ & 0.0 & $4.79 \pm 0.05$ & $\infty$ & 0.0 & Acro. \\
\hline 10 & $0.58 \pm 0.03$ & 0.0 & $0.58 \pm 0.03$ & $4.63 \pm 0.04$ & 0.0 & $4.63 \pm 0.04$ & $\infty$ & 0.0 & Acro. \\
\hline 11 & $0.57 \pm 0.03$ & 0.0 & $0.57 \pm 0.03$ & $4.55 \pm 0.04$ & 0.0 & $4.55 \pm 0.04$ & $\infty$ & 0.0 & Acro. \\
\hline 12 & $0.55 \pm 0.05$ & 0.0 & $0.55 \pm 0.05$ & $4.39 \pm 0.03$ & 0.0 & $4.39 \pm 0.03$ & $\infty$ & 0.0 & Acro. \\
\hline 13 & $0.53 \pm 0.04$ & 0.0 & $0.53 \pm 0.04$ & $4.23 \pm 0.05$ & 0.0 & $4.23 \pm 0.05$ & $\infty$ & 0.0 & Acro. \\
\hline 14 & $0.51 \pm 0.05$ & 0.0 & $0.51 \pm 0.05$ & $4.07 \pm 0.04$ & 0.0 & $4.07 \pm 0.04$ & $\infty$ & 0.0 & Acro. \\
\hline 15 & $0.49 \pm 0.05$ & 0.0 & $0.49 \pm 0.05$ & $3.91 \pm 0.03$ & 0.0 & $3.91 \pm 0.03$ & $\infty$ & 0.0 & Acro. \\
\hline 16 & $0.45 \pm 0.05$ & 0.0 & $0.45 \pm 0.05$ & $3.59 \pm .0 .03$ & 0.0 & $3.59 \pm .0 .03$ & $\infty$ & 0.0 & Acro. \\
\hline 17 & $0.42 \pm 0.05$ & 0.0 & $0.42 \pm 0.05$ & $3.35 \pm 0.03$ & 0.0 & $3.35 \pm 0.03$ & $\infty$ & 0.0 & Acro. \\
\hline 18 & $0.41 \pm 0.04$ & 0.0 & $0.41 \pm 0.04$ & $3.27 \pm 0.05$ & 0.0 & $3.27 \pm 0.05$ & $\infty$ & 0.0 & Acro. \\
\hline 19 & $0.39 \pm 0.03$ & 0.0 & $0.39 \pm 0.03$ & $3.12 \pm 0.04$ & 0.0 & $3.12 \pm 0.04$ & $\infty$ & 0.0 & Acro. \\
\hline 20 & $0.38 \pm 0.03$ & 0.0 & $0.38 \pm 0.03$ & $3.04 \pm 0.03$ & 0.0 & $3.04 \pm 0.03$ & $\infty$ & 0.0 & Acro. \\
\hline 21 & $0.37 \pm 0.03$ & 0.0 & $0.37 \pm 0.03$ & $2.95 \pm 0.04$ & 0.0 & $2.95 \pm 0.04$ & $\infty$ & 0.0 & Acro. \\
\hline 22 & $0.35 \pm 0.04$ & 0.0 & $0.35 \pm 0.04$ & $2.79 \pm 0.04$ & 0.0 & $2.79 \pm 0.04$ & $\infty$ & 0.0 & Acro. \\
\hline 23 & $0.34 \pm 0.04$ & 0.0 & $0.34 \pm 0.04$ & $2.71 \pm 0.05$ & 0.0 & $2.71 \pm 0.05$ & $\infty$ & 0.0 & Acro. \\
\hline 24 & $0.31 \pm 0.04$ & 0.0 & $0.31 \pm 0.04$ & $2.48 \pm 0.05$ & 0.0 & $2.48 \pm 0.05$ & $\infty$ & 0.0 & Acro. \\
\hline Sum. & & & $12.52 \pm 0.04$ & & & & & & \\
\hline
\end{tabular}

\section{Sparus aurata}

This species belongs to family sparidae. The photographed spreads of cells of this species (Fig.5) and karyotype showed the diploid chromosome number $(2 n=48)$ and all were acrocentric chromosomes, as illustrated in (Fig.5). The relative length of these chromosomes ranged from $1.91 \%$ to $5.83 \%$, centromeric indices were equal to zero and arm ratio of $\infty$ as illustrated in Table (3). 


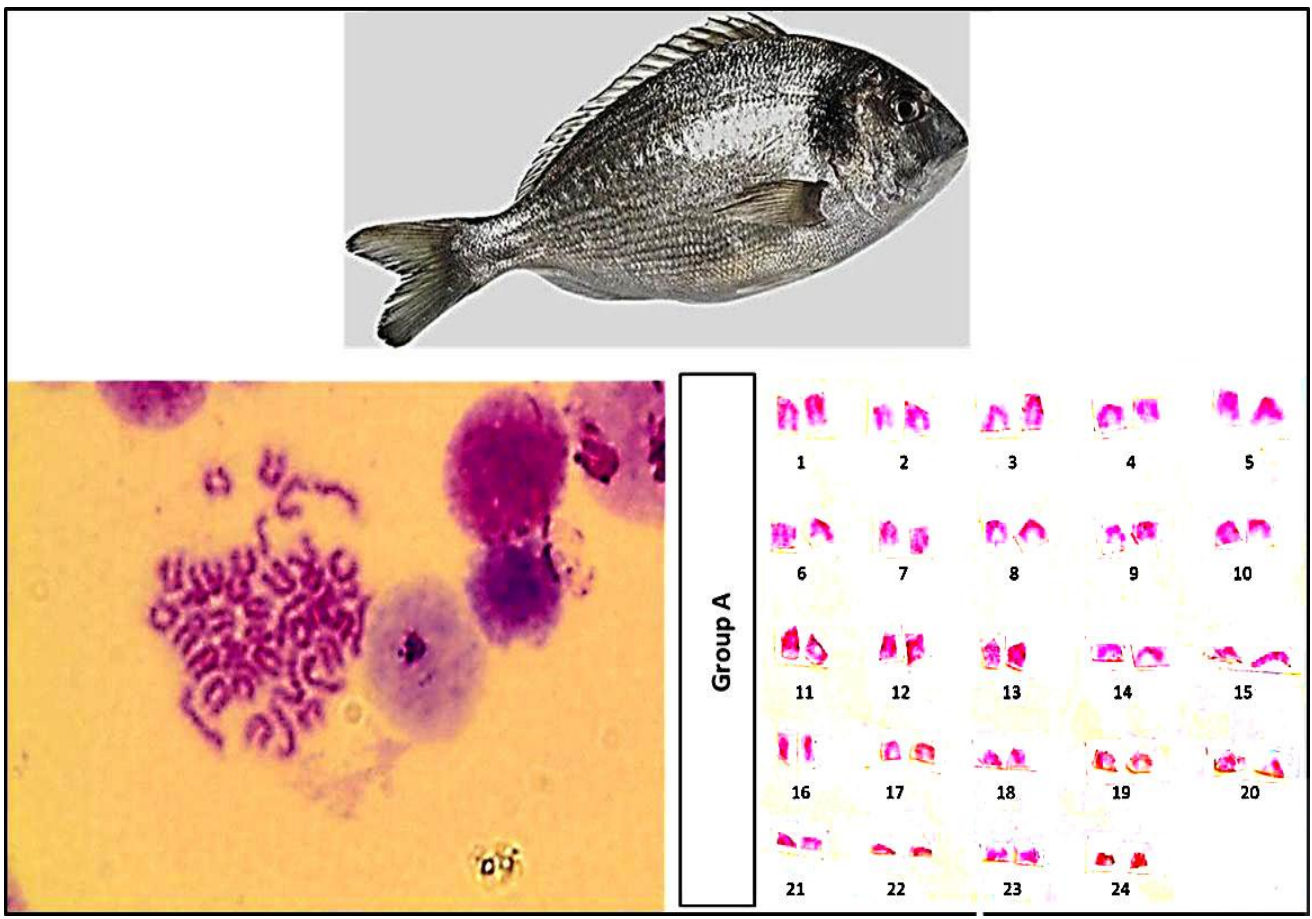

Fig. 5. A coloured photograph, chromosomes spread and karyotype of Sparus aurata.

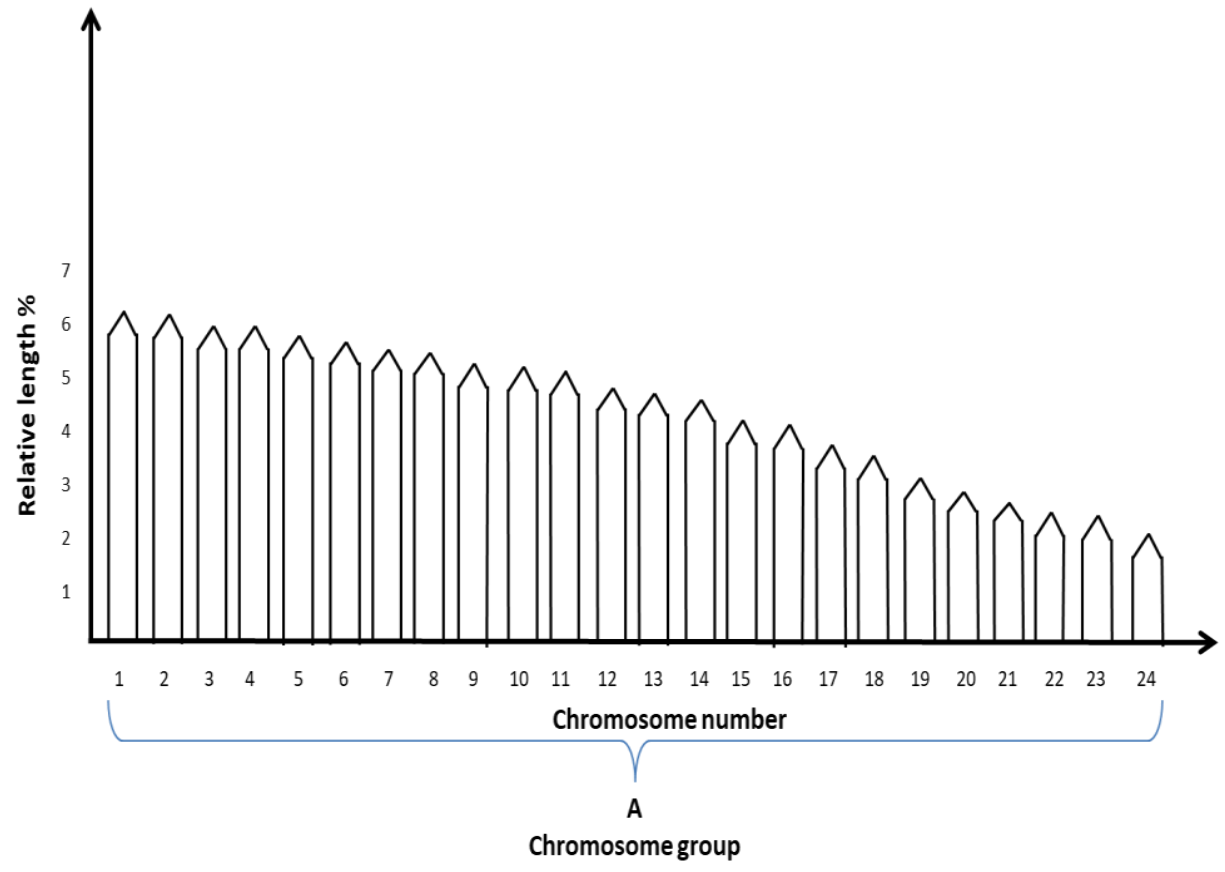

Fig. 6. Idiogram of chromosomes of Sparus aurata which constructed in respect to relative length. 
Table 3. Average of ten spreads of chromosomes measurements and classification of Sparus aurata.

\begin{tabular}{|c|c|c|c|c|c|c|c|c|c|}
\hline \multirow{3}{*}{ 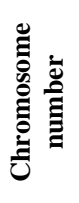 } & \multicolumn{3}{|c|}{ Chromosome length } & \multicolumn{3}{|c|}{ Relative length } & \multirow{2}{*}{$\begin{array}{l}\text { Arm } \\
\text { ratio }\end{array}$} & \multirow{2}{*}{$\begin{array}{l}\text { Centromeric } \\
\text { Index \% }\end{array}$} & \multirow{3}{*}{ 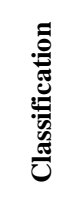 } \\
\hline & Long arm & $\begin{array}{l}\text { Short } \\
\text { arm }\end{array}$ & Total & Long arm & $\begin{array}{l}\text { Short } \\
\text { arm }\end{array}$ & Total & & & \\
\hline & Mean \pm S.D & $\begin{array}{l}\text { Mean } \pm \\
\text { S.D }\end{array}$ & Mean \pm S.D & Mean \pm S.D & $\begin{array}{l}\text { Mean } \pm \\
\text { S.D }\end{array}$ & Mean \pm S.D & $\begin{array}{l}\text { Mean } \pm \\
\text { S.D }\end{array}$ & Mean \pm S.D & \\
\hline 1 & $0.61 \pm 0.03$ & 0.0 & $0.61 \pm 0.03$ & $5.83 \pm 0.05$ & 0.0 & $5.83 \pm 0.05$ & $\infty$ & 0.0 & Acro. \\
\hline 2 & $0.60 \pm 0.04$ & 0.0 & $0.60 \pm 0.04$ & $5.73 \pm 0.03$ & 0.0 & $5.73 \pm 0.03$ & $\infty$ & 0.0 & Acro. \\
\hline 3 & $0.59 \pm 0.04$ & 0.0 & $0.59 \pm 0.04$ & $5.64 \pm 0.04$ & 0.0 & $5.64 \pm 0.04$ & $\infty$ & 0.0 & Acro. \\
\hline 4 & $0.58 \pm 0.05$ & 0.0 & $0.58 \pm 0.05$ & $5.54 \pm 0.05$ & 0.0 & $5.54 \pm 0.05$ & $\infty$ & 0.0 & Acro. \\
\hline 5 & $0.57 \pm 0.03$ & 0.0 & $0.57 \pm 0.03$ & $5.44 \pm 0.03$ & 0.0 & $5.44 \pm 0.03$ & $\infty$ & 0.0 & Acro. \\
\hline 6 & $0.56 \pm 0.03$ & 0.0 & $0.56 \pm 0.03$ & $5.35 \pm 0.04$ & 0.0 & $5.35 \pm 0.04$ & $\infty$ & 0.0 & Acro. \\
\hline 7 & $0.54 \pm 0.03$ & 0.0 & $0.54 \pm 0.03$ & $5.16 \pm 0.04$ & 0.0 & $5.16 \pm 0.04$ & $\infty$ & 0.0 & Acro. \\
\hline 8 & $0.53 \pm 0.05$ & 0.0 & $0.53 \pm 0.05$ & $5.06 \pm 0.04$ & 0.0 & $5.06 \pm 0.04$ & $\infty$ & 0.0 & Acro. \\
\hline 9 & $0.52 \pm 0.05$ & 0.0 & $0.52 \pm 0.05$ & $4.96 \pm 0.04$ & 0.0 & $4.96 \pm 0.04$ & $\infty$ & 0.0 & Acro. \\
\hline 10 & $0.51 \pm 0.05$ & 0.0 & $0.51 \pm 0.05$ & $4.87 \pm 0.05$ & 0.0 & $4.87 \pm 0.05$ & $\infty$ & 0.0 & Acro. \\
\hline 11 & $0.50 \pm 0.04$ & 0.0 & $0.50 \pm 0.04$ & $4.78 \pm 0.05$ & 0.0 & $4.78 \pm 0.05$ & $\infty$ & 0.0 & Acro. \\
\hline 12 & $0.47 \pm 0.05$ & 0.0 & $0.47 \pm 0.05$ & $4.48 \pm 0.03$ & 0.0 & $4.48 \pm 0.03$ & $\infty$ & 0.0 & Acro. \\
\hline 13 & $0.46 \pm 0.04$ & 0.0 & $0.46 \pm 0.04$ & $4.39 \pm 0.03$ & 0.0 & $4.39 \pm 0.03$ & $\infty$ & 0.0 & Acro. \\
\hline 14 & $0.45 \pm 0.03$ & 0.0 & $0.45 \pm 0.03$ & $4.29 \pm 0.03$ & 0.0 & $4.29 \pm 0.03$ & $\infty$ & 0.0 & Acro. \\
\hline 15 & $0.42 \pm 0.05$ & 0.0 & $0.42 \pm 0.05$ & $4.01 \pm 0.04$ & 0.0 & $4.01 \pm 0.04$ & $\infty$ & 0.0 & Acro. \\
\hline 16 & $0.40 \pm 0.04$ & 0.0 & $0.40 \pm 0.04$ & $3.82 \pm 0.03$ & 0.0 & $3.82 \pm 0.03$ & $\infty$ & 0.0 & Acro. \\
\hline 17 & $0.36 \pm 0.05$ & 0.0 & $0.36 \pm 0.05$ & $3.44 \pm 0.03$ & 0.0 & $3.44 \pm 0.03$ & $\infty$ & 0.0 & Acro. \\
\hline 18 & $0.33 \pm 0.05$ & 0.0 & $0.33 \pm 0.05$ & $3.15 \pm 0.05$ & 0.0 & $3.15 \pm 0.05$ & $\infty$ & 0.0 & Acro. \\
\hline 19 & $0.30 \pm 0.03$ & 0.0 & $0.30 \pm 0.03$ & $2.86 \pm 0.04$ & 0.0 & $2.86 \pm 0.04$ & $\infty$ & 0.0 & Acro. \\
\hline 20 & $0.28 \pm 0.03$ & 0.0 & $0.28 \pm 0.03$ & $2.67 \pm 0.03$ & 0.0 & $2.67 \pm 0.03$ & $\infty$ & 0.0 & Acro. \\
\hline 21 & $0.25 \pm 0.03$ & 0.0 & $0.25 \pm 0.03$ & $2.39 \pm 0.04$ & 0.0 & $2.39 \pm 0.04$ & $\infty$ & 0.0 & Acro. \\
\hline 22 & $0.23 \pm 0.04$ & 0.0 & $0.23 \pm 0.04$ & $2.19 \pm 0.03$ & 0.0 & $2.19 \pm 0.03$ & $\infty$ & 0.0 & Acro. \\
\hline 23 & $0.21 \pm 0.05$ & 0.0 & $0.21 \pm 0.05$ & $2.00 \pm 0.03$ & 0.0 & $2.00 \pm 0.03$ & $\infty$ & 0.0 & Acro. \\
\hline 24 & $0.20 \pm 0.03$ & 0.0 & $0.20 \pm 0.03$ & $1.91 \pm 0.05$ & 0.0 & $1.91 \pm 0.05$ & $\infty$ & 0.0 & Acro. \\
\hline Sum. & & & $10.47 \pm 0.05$ & & & & & & \\
\hline
\end{tabular}

\section{Pomadasys stridens}

This species belongs to family haemulidae. The photographed spreads of cells of this species (Fig.7) and karyotype showed the diploid chromosome number $(2 n=48)$ and all were acrocentric chromosomes, as illustrated in (Fig.7). The relative length of these 
chromosomes ranged from $2.73 \%$ to $5.61 \%$, centromeric indices were equal to zero and arm ratio of $\infty$ as shown in Table (4).

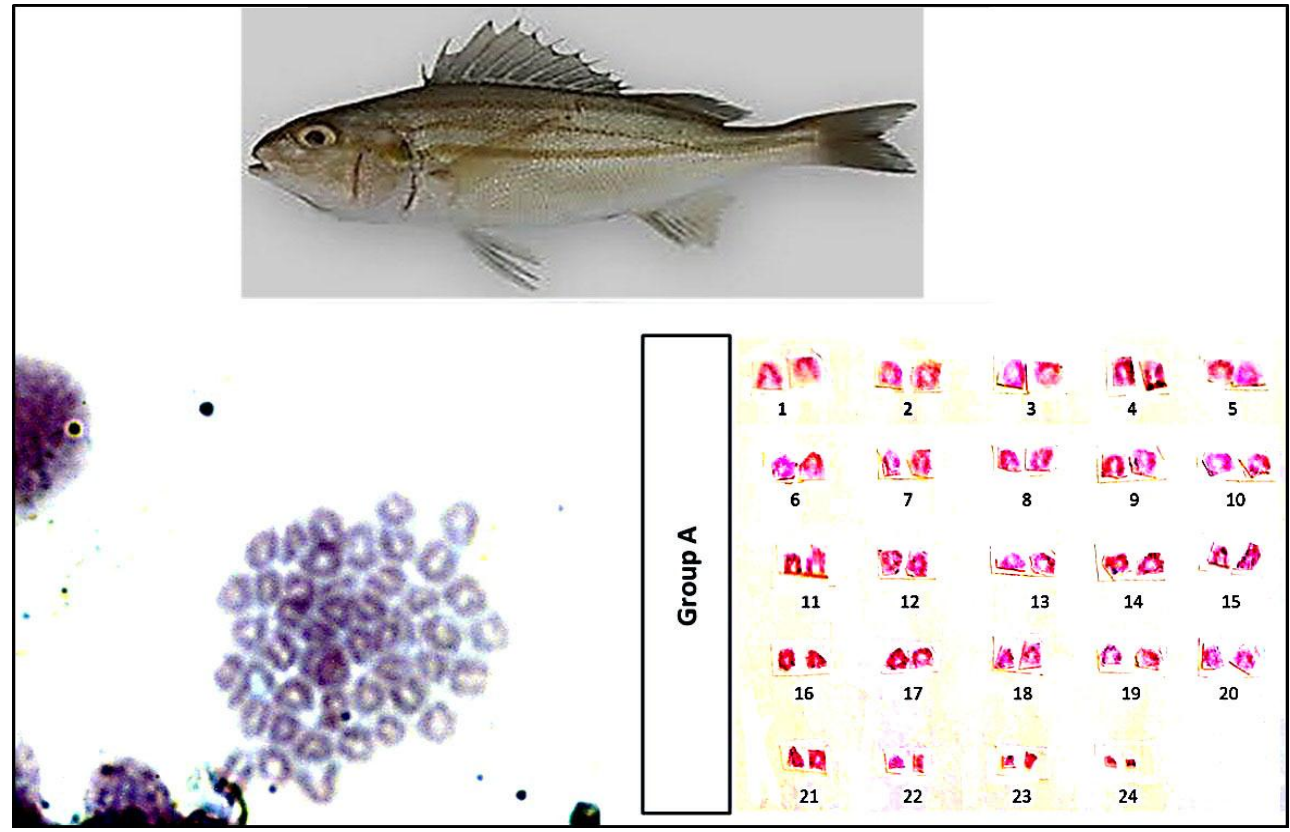

Fig. 7. A coloured photograph, chromosomes spread and karyotype of Pomadasys stridens.

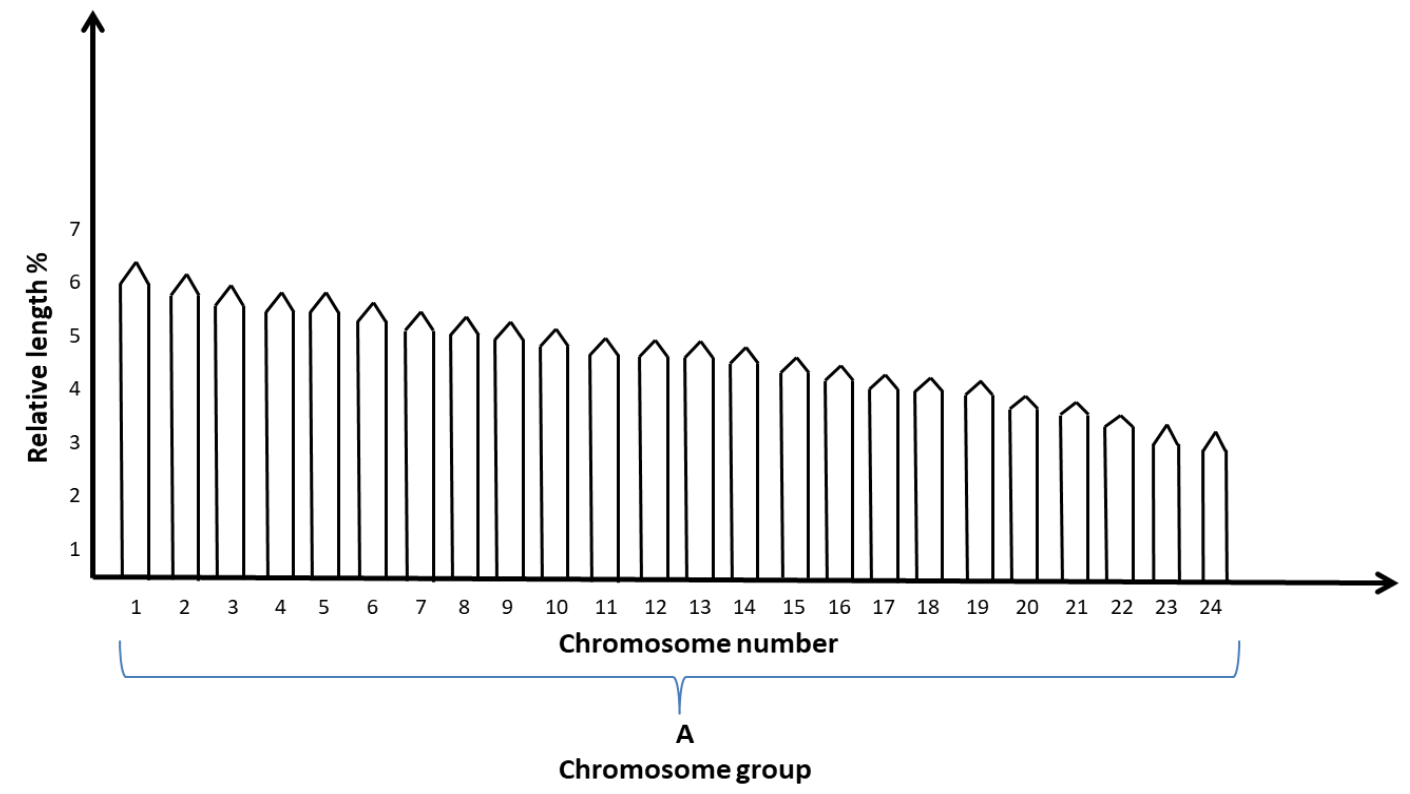

Fig. 8. Idiogram of chromosomes of Pomadasys stridens which constructed in respect to relative length. 
Ali H. Abu Almaaty et al., 2020

Table 4. Average of ten spreads of chromosomes measurements and classification of Pomadasys stridens.

\begin{tabular}{|c|c|c|c|c|c|c|c|c|c|}
\hline \multirow{3}{*}{ 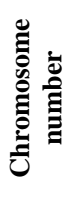 } & \multicolumn{3}{|c|}{ Chromosome length } & \multicolumn{3}{|c|}{ Relative length } & \multirow{2}{*}{$\begin{array}{l}\text { Arm } \\
\text { ratio }\end{array}$} & \multirow{2}{*}{$\begin{array}{l}\text { Centromeric } \\
\text { Index\% }\end{array}$} & \multirow{3}{*}{ 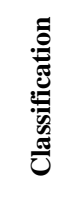 } \\
\hline & Long arm & $\begin{array}{l}\text { Short } \\
\text { arm }\end{array}$ & Total & Long arm & $\begin{array}{l}\text { Short } \\
\text { arm }\end{array}$ & Total & & & \\
\hline & Mean \pm S.D & $\begin{array}{l}\text { Mean } \pm \\
\text { S.D }\end{array}$ & Mean \pm S.D & Mean \pm S.D & $\begin{array}{l}\text { Mean } \pm \\
\text { S.D }\end{array}$ & Mean \pm S.D & $\begin{array}{l}\text { Mean } \pm \\
\text { S.D }\end{array}$ & Mean \pm S.D & \\
\hline 1 & $0.78 \pm 0.05$ & 0.0 & $0.78 \pm 0.05$ & $5.61 \pm 0.04$ & 0.0 & $5.61 \pm 0.04$ & $\infty$ & 0.0 & Acro. \\
\hline 2 & $0.76 \pm 0.04$ & 0.0 & $0.76 \pm 0.04$ & $5.47 \pm 0.03$ & 0.0 & $5.47 \pm 0.03$ & $\infty$ & 0.0 & Acro. \\
\hline 3 & $0.73 \pm 0.04$ & 0.0 & $0.73 \pm 0.04$ & $5.25 \pm 0.04$ & 0.0 & $5.25 \pm 0.04$ & $\infty$ & 0.0 & Acro. \\
\hline 4 & $0.72 \pm 0.03$ & 0.0 & $0.72 \pm 0.03$ & $5.19 \pm 0.05$ & 0.0 & $5.19 \pm 0.05$ & $\infty$ & 0.0 & Acro. \\
\hline 5 & $0.70 \pm 0.04$ & 0.0 & $0.70 \pm 0.04$ & $5.04 \pm 0.05$ & 0.0 & $5.04 \pm 0.05$ & $\infty$ & 0.0 & Acro. \\
\hline 6 & $0.69 \pm 0.05$ & 0.0 & $0.69 \pm 0.05$ & $4.97 \pm 0.05$ & 0.0 & $4.97 \pm 0.05$ & $\infty$ & 0.0 & Acro. \\
\hline 7 & $0.66 \pm 0.05$ & 0.0 & $0.66 \pm 0.05$ & $4.75 \pm 0.03$ & 0.0 & $4.75 \pm 0.03$ & $\infty$ & 0.0 & Acro. \\
\hline 8 & $0.64 \pm 0.03$ & 0.0 & $0.64 \pm 0.03$ & $4.61 \pm 0.04$ & 0.0 & $4.61 \pm 0.04$ & $\infty$ & 0.0 & Acro. \\
\hline 9 & $0.63 \pm 0.03$ & 0.0 & $0.63 \pm 0.03$ & $4.54 \pm 0.03$ & 0.0 & $4.54 \pm 0.03$ & $\infty$ & 0.0 & Acro. \\
\hline 10 & $0.62 \pm 0.04$ & 0.0 & $0.62 \pm 0.04$ & $4.47 \pm 0.05$ & 0.0 & $4.47 \pm 0.05$ & $\infty$ & 0.0 & Acro. \\
\hline 11 & $0.60 \pm 0.05$ & 0.0 & $0.60 \pm 0.05$ & $4.32 \pm 0.02$ & 0.0 & $4.32 \pm 0.02$ & $\infty$ & 0.0 & Acro. \\
\hline 12 & $0.59 \pm 0.04$ & 0.0 & $0.59 \pm 0.04$ & $4.25 \pm 0.05$ & 0.0 & $4.25 \pm 0.05$ & $\infty$ & 0.0 & Acro. \\
\hline 13 & $0.58 \pm 0.03$ & 0.0 & $0.58 \pm 0.03$ & $4.18 \pm 0.04$ & 0.0 & $4.18 \pm 0.04$ & $\infty$ & 0.0 & Acro. \\
\hline 14 & $0.55 \pm 0.05$ & 0.0 & $0.55 \pm 0.05$ & $3.96 \pm 0.04$ & 0.0 & $3.96 \pm 0.04$ & $\infty$ & 0.0 & Acro. \\
\hline 15 & $0.54 \pm 0.05$ & 0.0 & $0.54 \pm 0.05$ & $3.89 \pm 0.04$ & 0.0 & $3.89 \pm 0.04$ & $\infty$ & 0.0 & Acro. \\
\hline 16 & $0.53 \pm 0.04$ & 0.0 & $0.53 \pm 0.04$ & $3.82 \pm 0.03$ & 0.0 & $3.82 \pm 0.03$ & $\infty$ & 0.0 & Acro. \\
\hline 17 & $0.51 \pm 0.05$ & 0.0 & $0.51 \pm 0.05$ & $3.67 \pm 0.04$ & 0.0 & $3.67 \pm 0.04$ & $\infty$ & 0.0 & Acro. \\
\hline 18 & $0.50 \pm 0.03$ & 0.0 & $0.50 \pm 0.03$ & $3.60 \pm 0.04$ & 0.0 & $3.60 \pm 0.04$ & $\infty$ & 0.0 & Acro. \\
\hline 19 & $0.48 \pm 0.03$ & 0.0 & $0.48 \pm 0.03$ & $3.46 \pm 0.05$ & 0.0 & $3.46 \pm 0.05$ & $\infty$ & 0.0 & Acro. \\
\hline 20 & $0.45 \pm 0.02$ & 0.0 & $0.45 \pm 0.02$ & $3.24 \pm 0.03$ & 0.0 & $3.24 \pm 0.03$ & $\infty$ & 0.0 & Acro. \\
\hline 21 & $0.44 \pm 0.04$ & 0.0 & $0.44 \pm 0.04$ & $3.17 \pm 0.04$ & 0.0 & $3.17 \pm 0.04$ & $\infty$ & 0.0 & Acro. \\
\hline 22 & $0.40 \pm 0.05$ & 0.0 & $0.40 \pm 0.05$ & $2.88 \pm 0.03$ & 0.0 & $2.88 \pm 0.03$ & $\infty$ & 0.0 & Acro. \\
\hline 23 & $0.39 \pm 0.03$ & 0.0 & $0.39 \pm 0.03$ & $2.81 \pm 0.03$ & 0.0 & $2.81 \pm 0.03$ & $\infty$ & 0.0 & Acro. \\
\hline 24 & $0.38 \pm 0.04$ & 0.0 & $0.38 \pm 0.04$ & $2.73 \pm 0.05$ & 0.0 & $2.73 \pm 0.05$ & $\infty$ & 0.0 & Acro. \\
\hline Sum. & & & $13.88 \pm 0.04$ & & & & & & \\
\hline
\end{tabular}

\section{DISCUSSION}

This study presented cytogenetic information for four fish species (Argyrosomus regius of family Scianidae, Pomadasys stridens of family Haemulidae, Sparus aurata of family Scianidae and Dicentrarchus labrax of family Sparidae). There is a little information about the karyotype of these species. This study presented the differences in karyotype and idiograms of these four fish species. 
Most studies concluded that the diploid chromosome numbers of fishes ranging from $2 \mathrm{n}=16$ to $2 \mathrm{n}=134$. (Grassi et al., 2017, Abu Almaaty et al., 2015, 2017a \&2017b; Almeida et al., 2017).

The diploid chromosome numbers have a large diversity in species of fishes in Order perciformes which exhibit wide range, ranging from $2 n=20$ chromosomes in Pterolebias longipinnis (Rivulidae), up to $2 n=134$ in Corydoras aeneus (Calado, 2014). The diploid chromosome number of perciformes fish species was 48 chromosomes (Brum, 1995; Nirchio et al., 2014)

Our results are agree with results of (Nirchio et al., 2007; Accioly and Molina, 2008; Merlo et al., 2010; Neto et al., 2012; Nirchio et al., 2014; and Motta-Neto et al., 2019) which they reported that the diploid chromosomes number for four species under study was 48 chromosomes.

Karyological studies for determination of chromosome number and chromosomal formula of fish are of special interest to taxonomists because of the number of species and varieties of fish species have extreme diversity in their morphology.

\section{CONCLUSION}

Our results of this study indicated that the four species (Argyrosomus regius, Pomadasys stridens, Sparus aurata and Dicentrarchus labrax); have the same diploid chromosome number, chromosomal formula and fundamental number 48. Further studies are needed to different molecular techniques to investigate if some genes have specific sequence in each species.

\section{REFERENCES}

Abu-Almaaty, A. H.; Welson-Zekry, M. and Essa, Y. A. (2015). Using cytogenetic analysis RAPD in determination of genetic variations among four species of ornamental fishes of family: Poecilidae (Order: Cyprinodontiform). Genetika, 47(3), 1131-1148.

Abu-Almaaty, A. H.; Hassan M. K; Bahgat I. M. and Suleiman, M. E. E. (2017a). Karyological Characterization of Three Fish Species (Family: Characidae). Egyptian Journal of Aquatic Biology \& Fisheries, Vol. 21(1): 1-9.

Abu-Almaaty, A. H.; Hassan M. K.; Bahgat I. M. and Suleiman, M. E. E. (2017b). Inter Simple Sequence Repeat (ISSR) and Cytogenetic Analysis of Three Fish Species of Family Osphronemidae. Egyptian Journal of Aquatic Biology \& Fisheries, Vol. 21(2): 1-15.

Almeida, L. A.; Nunes, L. A.; Bitencourt, J. A.; Molina, W. F. and Affonso, P. R. (2017). Chromosomal Evolution and Cytotaxonomy in Wrasses (Perciformes; Labridae). J Hered. 108 (3): 239-253.

Accioly, I. V and Molina, W. F (2008). Cytogenetic studies in Brazilian marine Sciaenidae and Sparidae fishes (Perciformes), Genet. Mol. Res, 7 (2), PP. 358-370.

Bloom, D. D.; Weir, J. T.; Piller, K. R., and Lovejoy, N. R. (2013). Do freshwater fishes diversify faster than marine fishes? A test using state-dependent diversification analyses and molecular phylogenetics of New World silversides (Atherinopsidae). Evolution, 67(7), 2040-2057.

Brum, M. J. I. (1995). Correlações entre a filogenia ea citogenética dos peixes Teleósteos. Revista Brasileira de Genetica-Serie Monografias, 2, 5-42

Calado L.; Bertollo L. A. C; Cioffi . M. B.; Costa G. W. W. F.; Jacobina U. P. and Molina W. F. (2014). Evolutionary dynamics of rDNA genes on chromosomes of the 
Eucinostomus fishes: cytotaxonomic and karyoevolutive implications," Genetics and Molecular Research, 13 (4), pp. 9951-9959.

Eschmeyer, W. N. and Fong, J. D. (2014). Species by Family/Subfamily' Available at http://research.calacademy.org/research/ ichthyology/catalog/SpeciesByFamily.asp.

Grassi, D. J.; Swarça, A. C.; Dergam, J. A., Pastori, M. C., and Fenocchio, A. S. (2017). Cytogenetic characterization of Hoplias malabaricus (Bloch, 1794) from the Ctalamochita River (Córdoba, Argentina): first evidence for southernmost populations of this species complex and comments on its biogeography. Comparative Cytogenetics, 11 (1), 15-28.

King, R. C.; Stansfield, W. D. and Mulligen, P. K. (2006). A dictionary of genetics 7th ed', Oxford \& NY, P. 242.

Merlo, M. A.; Cross, I.; Chairi, H.; Manchado, M. and Rebordinos, L. (2010). Analysis of three multigene families as useful tools in species characterization of two closely-related species, Dicentrarchus labrax, Dicentrarchus punctatus and their hybrids. Genes \& genetic systems, 85(5), 341-349.

Molina W. F.; Neto C. M.; Sena D. C. S.; Cioffi, M. B. and Bertollo, L. A. C. (2012). Karyoevolutionary aspects of Atlantic hogfishes (Labridae-Bodianinae), with evidence of an atypical decondensed argentophilic heterochromatin. Marine genomics, 6, 25-31.

Molina W. F.; Da Costa G. W. W. F.; Soares R. X.; de Mello Affonso P. R. A.; de Bello Cioffi M.; and de Araújo W. C. et al. (2013). Extensive chromosome conservatism in Atlantic butterflyfishes, genus Chaetodon Linnaeus, 1758: Implications for the high hybridization success. Zoologischer Anzeiger-A Journal of Comparative Zoology, 253(2), 137-142.

Motta Neto, C. C.; Cioffi, M. D. B.; Costa, G. W. W. F.; Amorim, K. D. J.; Bertollo, L. A. C.; Artoni, R. F. and Molina, W. F. (2019). Overview on Karyotype Stasis in Atlantic Grunts (Eupercaria, Haemulidae) and the Evolutionary Extensions for Other Marine Fish Groups. Frontiers in Marine Science, 6, 628.

Neto, C. M.; Lima-Filho, P. A.; Araújo, W. C.; Bertollo, L. A. C. and Molina, W. F. (2012). Differentiated evolutionary pathways in Haemulidae (Perciformes): karyotype stasis versus morphological differentiation. Reviews in Fish Biology and Fisheries, 22(2), 457-465.

Neto, C. C. M.; Cioffi, M. B.; Bertollo, L. A. C. and Molina, W. F. (2011). Molecular cytogenetic analysis of Haemulidae fish (Perciformes): evidence of evolutionary conservation. Journal of Experimental Marine Biology and Ecology, 407(1), 97-100.

Netto M. R. D. C. B.; Pauls E. and de Mello Affonso, P. R. A. (2007). A standard protocol for obtaining fish chromosomes under post-mortem conditions. Micron, 38(3), 214-217.

Nelson, J. S. (2006). Fishes of the World, 4th Edition', New Jersey: John Wiley and Sons Inc.

Nirchio, M. and Cequea, H. (1998). Karyology of Mugil liza and M. curema from Venezuela', Bol. Inv. Mar. Cost., 27, pp. 45-50.

Nirchio, M.; Gaviria, J. I.; Oliveira, C.; Ferreira, I. A. and Martins, C. (2007). Cytogenetic analysis of three species of the genus Haemulon (Teleostei: Haemulinae) from Margarita Island, Venezuela. Genetica, 131(2), 135-140.

Nirchio, M.; Rossi, A. R.; Foresti, F. and Oliveira, C. (2014). Chromosome evolution in fishes: a new challenging proposal from Neotropical species, Neotropical Ichthyology, 12(4), PP. 761-770

O`Connor, C. (2008). Karyotyping for chromosomal abnormalities, Boston College', Nature Education, 1(1).

Reinhardt, R. (2014). European sea bass genome and its variation provide insights into adaptation to euryhalinity and speciation. Nature communications, 5(1), 1-10.

Shalaby, S. I.; Gupta, N.; Gupta, D. K. and Verma, V. K. (2020). Chromosomal Metaphase Spreads with G and NOR bandings of Fresh Water Teleost, Clarias batrachus. Egyptian Journal of Veterinary Sciences, 51(1), 61-67. 